\title{
Illusory Correlation for Body Type and Happiness: Covariation Bias and its Relationship to Eating Disorder Symptoms
}

\author{
Richard J. Viken, $\mathrm{PhD}^{1}$ * \\ Teresa A. Treat, $\mathrm{PhD}^{2}$ \\ Staci L. Bloom, BA ${ }^{1}$ \\ Richard M. McFall, PhD ${ }^{1}$
}

\author{
ABSTRACT \\ Objective: The current study investi- \\ gates covariation bias (illusory correla- \\ tion) in the perceived association \\ between happiness and body type, as \\ well as the association between covaria- \\ tion bias and eating disorder symptoms. \\ Method: Undergraduate women $(n=$ \\ 186) rated pictures of women on a vari- \\ ety of attributes, including happiness, \\ degree of overweight, and attractiveness. \\ Later, they were asked to judge the \\ level of covariation between these attri- \\ butes that was present in the stimuli \\ that they had rated. Participants \\ also completed the Eating Disorder \\ Examination-Self-Report Questionnaire \\ (EDE-Q).
}

Results: Participants reported that there was a negative association between weight and happiness in the stimuli that they had rated, even though the true correlation in the data was zero. This covariation bias was stronger among participants with higher levels of eating disorder symptoms.

Discussion: The results suggest a cognitive bias that may play a role in maintaining and enhancing concerns about shape and weight in symptomatic women. (c) 2005 by Wiley Periodicals, Inc.

Keywords: covariation bias; illusory correlation; body type; eating disorder

(Int J Eat Disord 2005; 38:65-72)

\section{Introduction}

Cognition has played a central role in theories of the etiology (Cooper, 1997; Fairburn, Shafran, \& Cooper, 1999; Vitousek \& Hollon, 1990) and treatment (Anderson \& Maloney, 2001; Fairburn \& Harrison, 2003; Wilson, Fairburn, Agras, Walsh, \& Kraemer, 2002) of eating disorders. These theories assert that individual differences in cognitive processing of information about weight, shape, food, and eating play an important role in the development and maintenance of bulimia, anorexia nervosa, and other eating disorders. Although much of the work in this area has relied on introspective assessment of cognition by self-report questionnaires (Cooper, Cohen-Tovée, Todd, Wells, \& Tovée, 1997), some recent work has focused on performance-based measures of cognition. For instance, studies of individual differences in performance on the emotional

\footnotetext{
Accepted 1 June 2004

*Correspondence to: Richard J. Viken, PhD, Department of Psychology, Indiana University, 1101 East 10th Street. Bloomington, IN 47405. E-mail: viken@indiana.edu

${ }^{1}$ Department of Psychology, Indiana University, Bloomington, Indiana

${ }^{2}$ Department of Psychology, Yale University, New Haven, Connecticut

Published online 21 June 2005 in Wiley InterScience (www.interscience.wiley.com). DOI: 10.1002/eat.20149 (C) 2005 Wiley Periodicals, Inc.
}

Stroop (Cooper, Anastasiades, \& Fairburn, 1992; Cooper \& Fairburn, 1994) and dichotic-listening (Schotte, McNally, \& Turner, 1990) paradigms suggest that eating disorder symptoms are associated with greater attention to body, weight, and eatingrelated words. Similarly, models of attention and classification adapted from cognitive science (Viken, Treat, Nosofsky, McFall, \& Palmeri, 2002) have shown that compared with controls, highsymptom women show strikingly different patterns of perception and decision-making when evaluating stimuli varying in body shape and facial affect. Attention weights from multidimensional scaling analyses showed that high-symptom women's perceptions were influenced more by body shape and less by affect than those of control participants, and these attentional differences were highly predictive of individual differences in decision-making in a later categorization task. Results from all of these cognitive performance paradigms implicate altered attentional focus as an important feature of the eating disorders.

Although differences in attention to particular features of the environment may be one way in which symptomatic individuals differ from controls, there are other aspects of attention and perception that may play a role in these disorders. Individuals also may differ in the degree to which they perceive covariation between the attributes of 
different stimuli or events, or between events and outcomes. For instance, several studies have shown that individuals who are higher in anxiety symptoms perceive higher levels of covariation between feared stimuli and aversive events (Tomarken, Mineka, \& Cook, 1989; Tomarken, Sutton, \& Mineka, 1995), even when the situation is structured so that there is little or no true covariation. A tendency to perceive covariation between feared stimuli and aversive events would be expected to magnify or maintain that fear, even under conditions when an unbiased perspective would show that the fear is not justified.

A similar covariation bias could play a role in the cognitive processes contributing to eating disorders. For instance, a bias to perceive covariation between thinness and positive outcomes or between heaviness and negative outcomes could serve to enhance and maintain maladaptive attitudes about weight, shape, and body image, even in the presence of objective data that are inconsistent with these attitudes. Such a covariation bias, or illusory correlation, would suggest that symptomatic women will experience the environment in a way that confirms their preexisting beliefs, even if the actual events and outcomes in their environment do not differ from those experienced by control women. Covariation biases are implied by most cognitive theories of eating disorders, and they are assessed more explicitly in at least one self-report measure of core beliefs and assumptions in the eating disorders (Cooper et al., 1997).

The current study is an investigation of individual differences in covariation bias among women differing in eating disorder symptoms. We asked a sample of undergraduate women to view a number of pictures of young women and to rate those pictures on a number of theoretically relevant attributes, including degree of overweight versus underweight, pleasingness of body shape, happiness, and attractiveness. The stimulus set was designed so that weight and happiness, in particular, would be uncorrelated. After the ratings, participants were asked to evaluate the covariation between these attributes in the stimuli that they had rated. Although the ratings task itself was explicit, participants did not know ahead of time that they would be asked to provide the later covariation estimates. Finally, participants completed the (EDE-Q, Eating Disorder Examination-Self-Report Questionnaire Fairburn \& Beglin, 1994), a questionnaire-based assessment of eating disorder symptoms. We hypothesized that participants would have a general tendency to report that the stimuli showed a negative correlation between weight and happiness, and to report a positive correlation between pleasing body shape and happiness, even if those correlations were not present in the data. We also hypothesized that this covariation bias would be stronger in women higher in eating disorders symptoms as measured by the EDE-Q.

\section{Method}

\section{Participants}

Participants were 186 undergraduate women who completed the experiment for partial course credit. Their mean age was $19.01(S D=1.8)$ years. The ethnic distribution was $85.6 \%$ White, $5.3 \%$ Asian, $4.3 \%$ Hispanic, $1.1 \%$ Black, and $0.5 \%$ Native American.

\section{Stimuli}

Stimuli were 370 images of undergraduate women. Fifty-eight paid models were recruited from the university population. Each model was photographed under standard conditions: at a fixed distance; with standard lighting; standing erect; facing the camera; in front of a fixed background; and in similar clothing (white short-sleeved t-shirt, black stretch pants, and white socks). Each model was photographed depicting a sad expression, a neutral expression, and a happy expression, resulting in 174 original photos. Some of the photos then were modified digitally to make the models appear to be thinner or heavier than the original picture. Thus, facial affect and apparent thinness were manipulated to create a stimulus set in which the two attributes were uncorrelated.

\section{Measures}

Stimulus Ratings. The stimuli were rated on four attributes, using 10-point scales anchored by descriptors at each end point: How happy is she? $(1=$ very sad, $10=$ very happy); How overweight is she? ( 1 = very underweight, $10=$ very overweight); How attractive is she? $(1=$ very unattractive, $10=$ very attractive $)$; How pleasing do you think her body shape is? ( $1=$ not pleasing at all, 10 = very pleasing). The order of the four ratings was randomized for each participant and each participant rated a different set of 75 stimuli (chosen randomly from the full set of 370) for each of the attributes. After the four randomly ordered attributes, the participants rated the stimuli on two additional attributes (likable and self-confident) being considered for use in future studies. Both of these attributes proved to be highly correlated with happiness, and they are not considered in the current article.

Perceived Associations. Several questions assessed participants' perceptions of covariation between the rated 
attributes. Instructions were as follows: "You have rated a large number of pictures of women on several dimensions. We would like to know if you noticed a connection between different characteristics of the women. Please rate the following scales concerning the connection or association between the different attributes of the women." The five questions asked were: "What was the association between how attractive the woman was and how happy she was?"; "What was the association between how attractive the woman was and whether she was overweight?"; "What was the association between how happy the woman was and whether she was overweight?"; "What was the association between how happy the woman was and how pleasing her shape was?"; and "What was the association between how attractive the woman was and how pleasing her shape was?" For each question, there were five response options that specified the direction and magnitude of covariation. For example, the response options for the association between happiness and weight were: "Happy women were almost always higher weight"; "Happy women tended to be higher weight"; "No association between happiness and weight"; "Happy women tended to be lower weight"; and "Happy women were almost always lower weight." Response options for the other questions were of the same form with different trait labels. The weight-happiness, shape-happiness, and weight-attractiveness associations were central to the current study. The other questions were chosen to provide a broader context for the participants, so that the focus of the study was not obvious, and to show that participants would be able to recognize covariation in the data when it was present. The perceived association items are somewhat unusual in that they are discrete rather than continuous. However, unlike most discrete variables, they have a true zero and a symmetric structure, so that average scores are meaningful. In the analyses of the perceived associations, we use both parametric (assigning numerical ratings of $1, .5,0,-.5$, and -1 ) and nonparametric approaches, looking for converging results.

Rating Associations. To parallel the perceived associations between weight and happiness, shape and happiness, and weight and attractiveness, we computed indices of individual differences in the actual covariation between those attributes in participants' ratings. First, for each participant, we computed the correlation between the participant's ratings for pairs of attributes across all stimuli on which the participant made both ratings. For instance, we computed correlations between the weight ratings and the happiness ratings that each participant gave to the stimuli that happened to be randomly selected for both of these attributes. These correlations provided an index of the degree to which an individual participant's judgments of one attribute were associated with that participant's judgments of another attribute. Because stimuli were chosen randomly for each rating task, the number of stimuli contributing to this correlation (i.e., the number selected randomly for both attributes) varied across participants, but the average number of stimuli was 15 . We computed these selfbased rating associations for weight-happiness, shapehappiness, and weight-attractiveness. We also computed for each participant, the correlation between the participant's 75 ratings of a given attribute and the average normative ratings given by the full sample for the same 75 stimuli for another attribute. These norm-based rating associations provide an index of the degree to which, for instance, variation in participant's happiness ratings could be predicted by normative weight ratings for the same stimuli. They have the advantage of being based on a much larger sample of ratings. The norm-based rating associations are analogous to the utilization coefficients (Macho, 1997) often used to evaluate the determinants of perceptual judgments in learning and categorization tasks. Norm based rating associations were computed for correlations between normative weight and participant-rated happiness and attractiveness, and between normative shape and participant-rated happiness. Rating associations involving weight ratings also were computed substituting weight-squared, to evaluate possible nonlinear effects that were suggested by the normative data. Self-based and norm-based rating associations were Fisher $z$ transformed before analyses.

EDE-Q. The EDE-Q (Fairburn \& Beglin, 1994) is a selfreport version of the Eating Disorder Examination structured diagnostic interview (Fairburn \& Cooper, 1993) that assesses the behavioral and attitudinal features of eating disorders. The EDE-Q has four subscales assessing Dietary Restraint, Weight Concern, Shape Concern, and Eating Patterns. The subscales have good internal consistency and test-retest reliability (Luce \& Crowther, 1999), and strong correlations with the same scales when assessed by the structured interview (Fairburn \& Beglin, 1994). In addition to the subscales, we computed a symptom count measure, which was the average of items assessing the number of objective binges, the number of subjective binges, and the number of the times the respondent used vomiting, laxatives, diuretics, or vigorous exercise as a means of controlling weight or shape in the last 28 days. For some analyses, we computed a global mean of the EDE-Q subscales, using a cutoff of 4.0 (see Fairburn \& Cooper, 1993) to define a high-EDE group.

\section{Procedure}

Participants completed an informed consent statement and then were taken to a private room and seated in front of a computer terminal. Stimulus ratings were completed first. A stimulus was depicted on the left side of 
the screen and a rating question (e.g. "How happy is she?") was on the right side, accompanied by a rating scale from 1 to 10 . Participants clicked on a number to indicate their answer, and another randomly chosen stimulus appeared. After the stimulus ratings, participants completed the perceived association items, and then the EDE-Q.

\section{Results}

Table 1 illustrates the normative characteristics of the stimulus set. For each stimulus and each attribute, we computed the average attribute rating across all participants rating that stimulus. Thus, Table 1 collapses across participants (by averaging across participants), making the 370 stimuli the units of analysis. In addition to the rated attributes, we computed an additional weight variable by centering the weight ratings (subtracting the mean score across all stimuli from the score for each stimulus) and squaring the resulting deviation scores. This computed variable reflects the degree to which the woman depicted in a stimulus deviates from the average weight rating. Table 1 shows that weight and weight-squared were uncorrelated with happiness across the 370 stimuli, reflecting one of the goals of stimulus development. Attractiveness and pleasingness of shape both were relatively independent of weight, but both had substantial correlations with weight-squared. The correlation with weight-squared reflects the fact that both very underweight and very overweight women received low ratings on pleasingness of shape. They also tended to receive lower ratings on attractiveness, although the relationship was less striking. Both shape and happiness had strong relationships with attractiveness. The remaining analyses use participants as the units of analysis, but the stimulus relationships reflected in Table 1 provide a useful context for those analyses.

\section{Perceived Associations}

Using the numerical coding from -1 to 1 , the mean value for the perceived association between

TABLE 1. Intercorrelations of rated attributes across 370 stimuli

\begin{tabular}{lrccc}
\hline & Weight & Weight-Squared & Shape & Affect \\
\hline Weight-squared & -.23 & & & \\
Shape & -.19 & -.72 & & \\
Affect & .00 & .01 & .07 & \\
Attractive & -.03 & -.39 & .71 & .33 \\
\hline
\end{tabular}

Note: Because the ratings are not entirely independent, standard significance levels will be biased. Under an assumption of independence any correlation of magnitude higher than .1 would be significant $p<.05$. weight and happiness ("What was the association between how happy the woman was and whether she was overweight") was 0.16 , which was significantly different from zero, $t(185)=-5.33, p<.001$, Cohen's $d=.39$. This indicates that participants perceived a negative association between weight and happiness, despite the absence of a true association between happiness and either weight or weight-squared in the normative ratings (Table 1). Overall, $42 \%$ of participants perceived a negative association between happiness and weight in the stimuli that they rated, whereas $15 \%$ reported a positive association. The mean value for the perceived association between happiness and shape was .20, indicating that participants in general perceived that the women with more pleasing shapes were happier. This value was significantly different from zero, $t(185)=7.34, p<.001$, Cohen's $d=.54$.

The mean value for the perceived association between weight and attractiveness was -.28 , which was significantly different from zero, $t(185)=$ $-7.87, p<.001$, Cohen's $d=.58$. Sixty-eight percent of the sample reported a negative association between these attributes (i.e., reported that attractive women either tended to be or almost always were lower in weight). In contrast, the correlation between weight and attractiveness in the stimulus norms was very small. However, there was a correlation in the stimulus norms between weightsquared and attractiveness (-.39). This reflected a nonlinear pattern in which attractiveness ratings tended to increase as weight increased to about the middle of the weight distribution, and then began to decrease as weight increased above the mean. The ascending portion of this relationship would be consistent with a positive perceived association, whereas the descending portion would be consistent with the negative perceived association that was reported by the subjects. The perceived associations between happiness and attractiveness $(M=.41)$, and between attractiveness and shape $(M=.47)$, also were significantly different from zero. However, because these associations merely reflect the strong empirical correlations that were anticipated, and observed, between these attributes in the stimulus set (Table 1), they are of interest only to provide a context for the first three perceived associations.

Table 2 shows the polyserial correlations of the three theoretically relevant perceived association items with the subscales and the symptom count measure from the EDE-Q. Table 2 shows that the Dietary Restraint subscale, the Eating Concerns subscale, and the symptom count had significant negative correlations with the perceived association 
TABLE 2. Correlations between symptom scales and perceived association scores

\begin{tabular}{|c|c|c|c|c|c|}
\hline & \multicolumn{5}{|c|}{ EDE-Q Subscales } \\
\hline & Restraint & Weight Concern & Shape Concern & Eating Concern & Symptom Count \\
\hline Weight-happy & $-.18^{*}$ & -.10 & -.08 & $-.16^{*}$ & $-.24 * *$ \\
\hline Shape-happy & .09 & $.19 *$ & .14 & $.15^{*}$ & .10 \\
\hline Weight-attractive & $-.28 * *$ & -.14 & $-.17^{*}$ & $-.17^{*}$ & $-.36^{* *}$ \\
\hline
\end{tabular}

Note: EDE-Q = Eating Disorder Examination-Self-Report Questionnaire. ${ }^{*} p<.05 .{ }^{* *} p<.01$.

between happiness and weight. Because the average perceived association was negative, this result means that higher Dietary Restraint, higher Eating Concern, and higher symptom counts predicted a stronger perception that the thin women were happier and heavy women were sadder. To describe these relationships further, we compared the global high-EDE group with the remaining sample on the numerical recode of the perceived association. The high-EDE group had significantly more negative perceived associations between weight and happiness $(M=-.40)$ than the remaining participants $(M=-.12), t(184)=3.23, p<.01, d=.69$. Twenty-five percent of the high-symptom group reported that "Happy women were almost always lower weight," whereas only $2 \%$ of the remaining sample gave that response. Sixty-seven percent of the high-symptom group gave one of the negative estimates of the weight-happiness relationship, compared with $38 \%$ in the remaining sample. A nonparametric chi-square comparison of the groups was significant $\left(\chi_{4}^{2}=26.68, p<.001\right)$. Correlations between the EDE measures and the perceived association between shape and happiness were somewhat weaker. Only Weight concern and Eating Concern were significantly correlated with more positive perceived associations between shape and happiness. Although the high-EDE group showed the expected pattern of a more positive perceived association between shape and happiness, the differences between the groups did not reach significance.

Higher scores on Dietary Restraint, Shape Concern, Eating Concern, and the symptom count significantly predicted more strongly negative estimates of the weight-attractiveness relationship. The high-EDE group had a significantly more negative perceived association between weight and attractiveness $(M=-.48)$ than the remaining participants $(M=-.25), t(183)=2.10, p<.05, d=.46$. Thirty percent of the high-symptom participants reported that "Attractive women were almost always lower weight," whereas only $4 \%$ of the remaining sample gave that response. The nonparametric comparison was also significant $\left(\chi_{4}^{2}=22.44\right.$, $p<.001)$. Although these results are consistent with the theoretical expectation that higher symptom women perceive a stronger connection between weight and attractiveness, they do not necessarily reflect an illusory correlation, given that there were nonlinear relationships between rated weight and rated attractiveness in the stimuli.

\section{Rating Associations}

In contrast to the results for the perceived association items, none of the rating associations were significantly correlated with the EDE-Q variables. When the high-EDE group was compared with the remaining sample, the $z$ to r-transformed mean rating associations for the two groups were very similar, and the pattern of averaged individual correlations in each group was similar to the normative correlations in Table 1. For instance, the norm-based rating association between weight and happiness was .01 in the high-EDE group and -.02 in the rest of the sample, and the norm-based rating association between shape and happiness was .08 in the high-EDE group and .06 in the remaining sample. $t$ tests revealed no significant differences between the groups for any of the rating associations. Thus, participants' post-hoc reports reflected the theoretically expected differences in perceived associations between high and low-symptom women, but this was not reflected in their actual ratings. EDE-Q scores were, however, significantly correlated with participants' average ratings of weight across the 75 stimuli that they rated, with higher EDE-Q scores associated with higher average ratings of weight. Average weight ratings correlated .30 with Dietary Restraint, .24 with Shape Concern, and .24 with Weight Concern (all $p s<.01$ ).

Normative data showed a correlation between weight-squared and both attractiveness and pleasingness of shape, indicating that, if stimuli were ordered by rated weight, then ratings of attractiveness and shape would follow an inverted U shape, with both very low-weight and very high-weight women receiving low scores, and with ratings increasing to a peak somewhere in between. It would be expected that the point in the weight distribution where attractiveness or shape ratings 
peak should differ for high and low-symptom women. Although the current study design does not allow a full evaluation of individual differences in this kind of "ideal point" or "unfolding' pattern," it was possible to conduct a simple analysis. We divided the 375 stimuli into deciles based on the normative weight ratings and then computed each participant's average ratings for attractiveness and shape across the stimuli falling into each decile. Because participants rated only 75 randomly chosen stimuli, the number of rated stimuli in a given decile could be quite small, so we computed a moving average of three deciles, assigning to each participant a score based on the weight-based decile where her ratings of attractiveness and pleasingness of shape peaked. Although individual differences in peak attractiveness decile were not related to EDE-Q scores, the decile of peak shape rating was correlated -.24 with Dietary Restraint, -.19 with Eating Concern, -.21 with Shape Concern, and -.20 with symptom count (all $p$ s $<.01$ ). Thus, participants with higher levels of eating problems had pleasingness of shape ratings that peaked at a lower weight than low-symptom women. The pleasingness of shape ratings for the high-EDE group peaked at a significantly lower mean weight decile $(\mathrm{M}=2.50$, approximately the 25th percentile of the weight distribution) than the low-symptom group ( $\mathrm{M}=3.29$, approximately the 33rd percentile of the weight distribution), $t(184)=$ $3.34, p<.01, d=.71$. These relationships provide intriguing clues about nonlinear relationships to be pursued in future research.

\section{Discussion}

Participants in the current study perceived a significant negative association between weight and happiness and a significant positive association between pleasingness of shape and happiness in the stimuli that they rated, even though the attributes actually were uncorrelated in the stimuli. Further, perceived associations between weight and happiness were significantly correlated with the EDE-Q Dietary Restraint subscale and with an EDE-Q-based symptom count. Participants classified as high symptom by a global cut off on the EDE-Q were twice as likely to report a negative association between weight and happiness as were lower-symptom participants, and they were 10 times as likely to report that happy women in the stimuli were almost always of lower weight. The size of the effect in this group comparison $(d=.69)$ was substantial. Thus, subjects in general tended to report an illusory correlation between weight and happiness, and this perception was exaggerated among women with higher levels of eating disorder symptoms. If similar processes operate outside the laboratory, then we would expect symptomatic women to draw different conclusions about the relationship between weight and negative outcomes, even after experiencing the same events as lowsymptom women. Such a process could help to maintain or enhance maladaptive attitudes about weight, shape, and eating. Further, studies from cognitive science have shown that initial perceptual processes have broad implications for "downstream" cognitive processes such as categorization, leaning, decision-making, and memory (Ashby \& Gott, 1988; Kruschke \& Johansen, 1999; Nosofsky, 1992).

The fact that participants reported opposite, but theoretically consistent, associations between weight and happiness versus pleasingness of shape and happiness suggests that this illusory correlation effect cannot be explained by general factors causing a positive bias in correlation judgments based on small samples (Kareev, 1995). Several underlying processes could explain the phenomenon. Illusory correlation could reflect a strong previous expectation that is not effectively challenged by exposure to the true relationship in a set of stimuli. However, a strong previous expectation might have been expected to influence covariation in participants' actual ratings of weight and happiness, and we found no evidence that high-symptom women showed greater covariation in their explicit ratings. A second possibility is that the processing differences affect the implicit learning that takes place during the rating task, but that those processes are not reflected in the explicit ratings themselves. Finally, the differences in perceived covariation could reflect a memory bias that affects perceptions later, at the time that the perceived associations are estimated, not during the rating task.

A number of extensions to the current study would be helpful in distinguishing among these specific processes. Assessment of previous covariation expectancies, as well as manipulation of covariation expectancies (de Jong, Merckelbach, \& Arntz, 1995), would help to clarify their role. It also would be useful to explore a wider range of true covariation levels. In the current study, we considered only a stimulus sample where we expected the correlation between happiness and both weight and shape to be close to zero. A useful extension of the current study would be to provide participants with structured subsamples of stimuli in 
which the covariation between weight and happiness is manipulated. Although the current study provided information about individual differences in perceived association between happiness and body type when the true correlation was near zero, it would be of interest to know whether the highsymptom participants also inflate modest positive associations between thinness and happiness, and whether they fail to recognize modest negative associations between thinness and happiness. Participants in the current study did not know ahead of time that they would be asked to make covariation judgments, so it also would be useful investigate the effects of explicit instructions before the stimulus ratings.

With regard to the issue of perception versus memory, there are several procedures that allow on-line estimates of covariation bias during exposure to the stimuli. These procedures are less demanding of memory processes. For instance, it is possible to show participants a brief series of stimuli that differ in two attributes, such as facial affect and body type, and then show them a stimulus that has information about one attribute only, asking them to make a prediction about the other attribute (Kareev, Lieberman, \& Lev, 1997). It also is possible to estimate perceived covariation indirectly through multidimensional scaling analyses of similarity judgments (Treat et al., 2002) or by fitting models to simultaneous categorization data (Maddox \& Ashby, 1996).

Participants in the current study also reported that there was a negative relationship between weight and atractiveness in the stimuli that they had rated, but this may not be an illusory relationship in this stimulus set if the nonlinear association between weight and attractiveness is taken into account. Whether these perceptions should be considered illusory or not, the perceptions of highsymptom women showed an exaggerated perception of this relationship.

Although the methods used to evaluate covariation bias for weight and happiness in the current study were quite different from those used in the earlier literature on illusory correlation in high-fear individuals (Tomarken et al., 1989, 1995), the results are quite consistent with that work. It is possible that altered perceptions of covariation reflect a maladaptive process that is shared across a wide array of psychopathologic conditions. Because of the way in which perceptual processes influence downstream cognitive activity, differences in perception of covariation would have broad implications for problems in decision-making, mood regulation, and social competence that are associated with these disorders, and covariation bias may be worth evaluating as a target for cognitive interventions.

\section{References}

Anderson, D.A., \& Maloney, K.C. (2001). The efficacy of cognitivebehavioral therapy on the core symptoms of bulimia nervosa. Clinical Psychology Review, 21(7), 971-988.

Ashby, F.G., \& Gott, R.E. (1988). Decision rules in the perception and categorization of multidimensional stimuli. Journal of Experimental Psychology: Learning, Memory, and Cognition, 14, 33-53.

Cooper, M.J. (1997). Cognitive theory in anorexia nervosa and bulimia nervosa: A review. Behavioral and Cognitive Psychotherapy, 25, 113-145.

Cooper, M.J., Anastasiades, P., \& Fairburn, C.G. (1992). Selective processing of eating, shape-, and weight-related words in persons with bulimia nervosa. Journal of Abnormal Psychology, 101, 352-355.

Cooper, M.J., \& Fairburn, C.G. (1994). Changes in selective information processing with three psychological treatments for bulimia nervosa. British Journal of Clinical Psychology, 33, 353-356.

Cooper, M.L., Cohen-Tovée, E., Todd, G., Wells, A., \& Tovée, M. (1997). The eating disorder belief questionnaire: Preliminary development. Behaviour Research and Therapy, 35, 381-388.

de Jong, P.J., Merckelbach, H., \& Arntz, A. (1995). Covariation bias in phobic women: The relationship between a priori expectancy, on line expectancy, autonomic processing and a posteriori contingency judgement. Journal of Abnormal Psychology, 104, 52-62.

Fairburn, C.G., \& Beglin, S.J. (1994). Assessment of eating disorders: Interview or self-report questionnaire? International Journal of Eating Disorders, 16, 363-370.

Fairburn, C.G., \& Cooper, Z. (1993). The Eating Disorder Examination (12th ed.). In C.G. Fairburn \& G.T. Wilson (Eds.), Binge eating: Nature, assessment, and treatment (pp. 317-360). New York: Guilford Press.

Fairburn, C.G., \& Harrison, P.J. (2003). Eating disorders. Lancet, 361(9355), 407-416.

Fairburn, C.G., Shafran, R., \& Cooper, Z. (1999). A cognitive behavioural theory of anorexia nervosa. Behaviour Research and Therapy, 37, 1-13.

Kareev, Y. (1995). Positive bias in the perception of covariation. Psychological Review, 102(3), 490-502.

Kareev, Y., Lieberman, I., \& Lev, M. (1997). Through a narrow window: Sample size and the perception of correlation. Journal of Experimental Psychology: General, 126, 278-287.

Kruschke, J.K., \& Johansen, M.K. (1999). A model of probabilistic category learning. Journal of Experimental Psychology: Learning, Memory, and Cognition, 25, 1083-1119.

Luce, K.H., \& Crowther, J.H. (1999). The reliability of the Eating Disorder Examination-Self-Report Questionnaire Version (EDE-Q). International Journal of Eating Disorders, 25, 349-351.

Macho, S. (1997). Effect of relevance shifts in category acquisition: A test of neural networks. Journal of Experimental Psychology: Learning Memory and Cognition, 23, 30-53.

Maddox, W.T., \& Ashby, F.G. (1996). Perceptual separability, decisional separability, and the identification-speeded classification relationship. Journal of Experimental Psychology: Human Perception and Performance, 22, 795-817.

Nosofsky, R.M. (1992). Exemplar-based approach to relating categorization, identification, and recognition. In F.G. Ashby (Ed.), Multidimensional models of perception and cognition (pp. 363-393). Hillsdale, NJ: Erlbaum. 
Schotte, D.E., McNally, R.J., \& Turner, M.L. (1990). A dichotic listening analysis of body weight concern in bulimia nervosa. International Journal of Eating Disorders, 9 109-113.

Tomarken, A.J., Mineka, S., \& Cook, M. (1989). Fear-relevant selective associations and covariation bias. Journal of Abnormal Psychology, 98, 381-394.

Tomarken, A.J., Sutton, S.K., \& Mineka, S. (1995). Fear-relevant illusory correlations: What types of associations promote judgmental bias? Journal of Abnormal Psychology, 104, 312-326.

Treat, T.A., McFall, R.M., Viken, R.J., Nosofsky, R.M., MacKay, D.B., \& Kruschke, J.K. (2002). Assessing clinically relevant perceptual organization with multidimensional scaling techniques. Psychological Assessment, 14(3), 239-252.

Viken, R.J., Treat, T.A., Nosofsky, R.M., McFall, R.M., \& Palmeri, T.J. (2002). Modeling individual differences in perceptual and attentional processes related to bulimic symptoms. Journal of Abnormal Psychology, 111, 598-609.

Vitousek, K.B., \& Hollon, S.D. (1990). The investigation of schematic content and processing in eating disorders. Cognitive Therapy and Research, 14, 191-214.

Wilson, G.T., Fairburn, C.C., Agras, W.S., Walsh, B.T., \& Kraemer, H. (2002). Cognitive behavioral therapy for bulimia nervosa: Time course and mechanisms of change. Journal of Consulting and Clinical Psychology, 70(2), 267-274. 